\title{
RECONSTRUCTIVE UROLOGY
}

\author{
Surgical complications following radical cystectomy and orthotopic neobladders in women \\ Ali-el-Dein B, Shaaban AA, Abu-Eideh RH, el-Azab M, Ashamallah A, Ghoneim MA \\ Urology and Nephrology Center, Faculty of Medicine, Mansoura University, Mansoura, Egypt \\ J Urol. 2008; 180: 206-10; discussion 210
}

Purpose: Orthotopic neobladders have become the standard of care after radical cystectomy in select women with bladder cancer. We report early and late complications in 192 patients. Although medical complications were important, they were not the focus of this study.

Materials and Methods: Between January 1995 and December 2003, 192 women with a mean age of 50.6 years received an orthotopic neobladder after radical cystectomy for bladder cancer. Standard radical cystectomy was done. Ileal reservoirs were used, mostly in the form of an ileal W-neobladder. We evaluated the patients for functional outcome, early and late complications, and treatment for these complications.

Results: Two patients (1\%) died of pulmonary embolism 1 to 2 weeks after cystectomy. Followup was 6 to 125 months (mean 54). Early complications included hemorrhage requiring reexploration in 1 case, postoperative blood transfusion in 1 , wound infection in 8 , prolonged ileus in 5, deep vein thrombosis in 5, pouch-vaginal fistula in 6, prolonged urinary leakage in 3, pouch-cutaneous fistula in 1 and early ureteral obstruction in 1 . Of the 177 patients eligible for functional evaluation 62 experienced a total of 75 late complications, including stone disease in 18, ureteroileal stricture in 19, reflux in 22, intestinal obstruction in 2, incisional hernia in 2 and chronic pyelonephritis in 12. Early and late complications were treated accordingly with good outcomes. Conclusions: Early and late complications develop in a significant number of patients. Most early complications may be treated conservatively, while late complications are mostly treated with endourological and/or open surgery. Close lifelong surveillance of patients is mandatory to detect and properly treat these complications.

\section{Editorial Comment}

Bladder cancer and its treatment with the specific anatomical aspect became a growing issue for women in recent years (1). The ileum conduit and the continent cutaneous pouch seemed to be the only option for female patients; whereas the ileum orthotopic neobladder was already the common surgical treatment for males. In the female, the different anatomical continence mechanism within the muscular pelvis had to be considered. A solution came from one of the centers with a high cystectomy frequency for both sexes where the orthotopic neobladder was performed in the early phase (2).

Ali-El-Dein B et al. (3) published the short and long-term follow-up of 192 women who received a orthotopic ileum neobladder during the time period 1995 - 2003. The complications were primarily noted in the upper urinary tract and mostly caused by implantation strictures within the first two years after the surgery. The complications experienced are similar to others, independent to the sex. As recently noted by the Consensus Conference on Bladder Cancer of the World Health Organization (WHO), this issue cannot be solved by a single suggestion (4).

Increased knowledge of the pelvic anatomy helped to reduce, as the authors stated, the frequency of neobladder-vaginal fistula described by Stenzl (5). Although the presented data includes those with the technique of orthotopic neobladder of an early period, the continence rate is similar to others (6).

The growing anatomical knowledge results in better intraoperative identification and preservation of the sphincteric area and neurovascular structures thus improving the long-term outcome and satisfaction (7).

\section{References}

1. Stenzl A: Current Concepts for Urinary Diversion in Women. EAU-EBU, Update Series. 2003; 1: 91-9. 
2. Abol-Enein H, Ghoneim MA: Functional results of orthotopic ileal neobladder with serous-lined extramural ureteral reimplantation: experience with 450 patients. J Urol. 2001; 165: 1427-32.

3. Ali-el-Dein B, Shaaban AA, Abu-Eideh RH, el-Azab M, Ashamallah A, Ghoneim MA: Surgical complications following radical cystectomy and orthotopic neobladders in women. J Urol. 2008; 180: 206-10; discussion 210.

4. Hautmann RE, Gschwend JE, de Petriconi RC, Kron M, Volkmer BG: Cystectomy for transitional cell carcinoma of the bladder: results of a surgery only series in the neobladder era. J Urol. 2006; 176: 486-92; discussion 491-2.

5. Stenzl A, Nagele U, Kuczyk M, Sievert KD, Anastasiadis A, Seibold J: et al.: Cystectomy - Technical Considerations in Male and Female Patients. EAU-EBU, Update Series, 2005; 3: 138-46.

6. Nagele U, Kuczyk M, Anastasiadis AG, Sievert KD, Seibold J, Stenzl A: Radical cystectomy and orthotopic bladder replacement in females. Eur Urol. 2006; 50: 249-57.

7. Schilling D, Horstmann M, Nagele U, Sievert KD, Stenzl A: Cystectomy in women. BJU Int. 2008; 102: 1289-95.

\author{
Dr. Karl-Dietrich Sievert \& \\ Dr. Arnulf Stenzl \\ Department of Urology \\ Eberhard-Karls-University Tuebingen \\ Tuebingen, Germany \\ E-mail: arnulf.stenzl@med.uni-tuebingen.de
}

\title{
Apoptosis and effects of intracavernous bone marrow cell injection in a rat model of postprostatectomy erectile dysfunction \\ Fall PA, Izikki M, Tu L, Swieb S, Giuliano F, Bernabe J, Souktani R, Abbou C, Adnot S, Eddahibi S, Yiou R \\ Urology Department, Henri Mondor Teaching Hospital, Créteil, France; INSERM Unit 841, Teams 8 and 10, \\ plateforme du petit animal, School of Medicine, Henri Mondor Teaching Hospital, Créteil, France
}

Eur Urol. 2008; 9. [Epub ahead of print]

Objectives: To investigate the pathophysiology of postprostatectomy erectile dysfunction (pPED) in a rat model of bilateral cavernous nerve ablation (BCNA) and to assess the effects of local bone marrow mononuclear cell (BMMNC) injection on erectile dysfunction (ED) and cavernosal cellular abnormalities caused by BCNA. Design, Setting, and Participants: This was an experimental study in Fisher rats with BCNA.

Intervention: Intervention included BNCA, electrical stimulation of the pelvic ganglion, and local BMMNC injection.

Measurements: Erectile responses to electric pelvic ganglion stimulation were studied. Cavernous tissue was examined to determine the cell types undergoing apoptosis and to detect changes in protein and gene expression of neuronal nitric oxide synthase (nNOS) and endothelial nitric oxide synthase (eNOS) using real-time quantitative polymerase chain reaction (RTQ-PCR) and Western blotting. The effects of local BMMNC injection on these parameters were studied.

Results and Limitations: Diffuse apoptosis was noted in the connective tissue mesenchymal cells and vascular smooth muscle and endothelial cells. Compared with sham-operated controls, nNOS and eNOS levels were decreased after $3 \mathrm{wk}$ and were normal (eNOS) or increased (nNOS) after $5 \mathrm{wk}$, suggesting spontaneous nerve regeneration. Despite nNOS recovery, erectile responses to electrical stimulation remained impaired after $5 \mathrm{wk}$, when mesenchymal cell apoptosis was the main persistent biologic abnormality. BMMNC injection decreased apoptotic cell numbers, accelerated the normalisation of nNOS and eNOS, and partially restored erectile responses at week 5 .

Conclusions: Massive cell apoptosis may play a key role in the pathophysiology of pPED. In this animal model, apoptosis persisted despite spontaneous nerve regeneration, suggesting that the course of BCNA-induced cell 
dysfunction was independent of reinnervation. BMMNC improved erectile function by inhibiting apoptosis and may hold promise for repairing penile cell damage caused by radical prostatectomy (RP).

\title{
Editorial Comment
}

Erectile dysfunction, which is a result of apoptosis caused by bilateral cavernous nerve ablation, is probably a "worst case scenario" after a radical prostatectomy (1). Even though human nerves cover the prostate surface similar to a net, post-operative erectile dysfunction can occur after an intended nerve sparing (2). The function of this net-like distribution was intraoperatively verified by Kaiho et al. (3). Although impotence following radical prostatectomy is multi-factorial, neurogenic factors also seem to play a major role. The most important prognostic factors for sexual potency recovery after radical prostatectomy are the number of spared nerve fibers, age, and sexual activity prior the surgery.

Fall and colleagues found in their rat model that bilateral cavernous nerve ablation causes apoptosis predominately in the vimentin $+/ \alpha$-actin cells and is present throughout the cavernosal bodies, similar to the smooth muscle and the endothelial cells of the cavernosal arteries. With the intracavernosal delivery of bonemarrow mononucleated cells, apoptotic cells will be replaced to recover erectile function (1). This treatment strategy may constitute a promising alternative or complement treatments aimed at stimulating nerve regeneration similar to the recently reported testis stem cells that were differentiated into cells of all three germ layers (4).

The findings to protect corporal function noted by Fall et al. are not only relevant as a possible treatment immediately after radical prostatectomy, but also may be important for the aging but still sexually active patient. The improved knowledge of the nerve concourses will help to protect function [Sievert et al. Urology 2009, accepted for publication; scheduled for publication in March 2009] which might additionally minimize the erectile dysfunction with intracorporal injected bone-marrow mononucleated cells (2).

\section{References}

1. Fall PA, Izikki M, Tu L, Swieb S, Giuliano F, Bernabe J, et al.: Apoptosis and Effects of Intracavernous Bone Marrow Cell Injection in a Rat Model of Postprostatectomy Erectile Dysfunction. Eur Urol. 2008; 9. [Epub ahead of print]

2. Sievert KD, Hennenlotter J, Laible I, Amend B, Schilling D, Anastasiadis A, et al.: The periprostatic autonomic nervesbundle or layer? Eur Urol. 2008; 54: 1109-17.

3. Kaiho Y, Nakagawa H, Saito H, Ito A, Ishidoya S, Saito S, et al.: Nerves at the Ventral Prostatic Capsule Contribute to Erectile Function: Initial Electrophysiological Assessment in Humans. Eur Urol. 2008; 24. [Epub ahead of print]

4. Conrad S, Renninger M, Hennenlotter J, Wiesner T, Just L, Bonin M, et al.: Generation of pluripotent stem cells from adult human testis. Nature. 2008; 456: 344-9.

\author{
Dr. Karl-Dietrich Sievert \& \\ Dr. Arnulf Stenzl \\ Department of Urology \\ Eberhard-Karls-University Tuebingen \\ Tuebingen, Germany \\ E-mail: arnulf.stenzl@med.uni-tuebingen.de
}

